\title{
Téoros
}

Revue de recherche en tourisme

\section{La formation de la relève en tourisme au Saguenay-Lac-Saint-Jean}

\section{Réjean Côté}

Volume 19, numéro 1, printemps 2000

Pour une culture du tourisme au Saguenay-Lac-Saint-Jean

URI : https://id.erudit.org/iderudit/1071804ar

DOI : https://doi.org/10.7202/1071804ar

Aller au sommaire du numéro

Éditeur(s)

Université du Québec à Montréal

ISSN

0712-8657 (imprimé)

1923-2705 (numérique)

Découvrir la revue

Citer cet article

Côté, R. (2000). La formation de la relève en tourisme au

Saguenay-Lac-Saint-Jean. Téoros, 19(1), 47-49.

https://doi.org/10.7202/1071804ar d'utilisation que vous pouvez consulter en ligne.

https://apropos.erudit.org/fr/usagers/politique-dutilisation/ 


\section{LA FORMATION DE LA RELÈVE EN TOURISME AU SAGUENAY-LAC-SAINT-JEAN}

\section{Réjean Côté}

Aujourd'hui, plus que jamais, l'industrie touristique doit s'appuyer sur sa principale richesse, les ressources humaines, pour assurer sa consolidation et sa croissance. L'élément humain des organisations constitue la pierre angulaire de l'ambitieux projet de mobilisation de tous les effectifs vers l'atteinte du même but : le succès. Qui plus est, la ressource humaine doit être de qualité, d'une compétence éprouvée, conforme aux nouveaux standards d'excellence, polyvalente et résolument orientée vers la satisfaction de la clientèle. La ressource humaine, comme d'ailleurs les organisations qui l'emploient, doit désormais évoluer en ayant toujours le touriste au centre de ses préoccupations : combler les besoins en faisant vivre une "expérience touristique 》 réussie. Nous décrirons dans le présent article l'évolution de la formation de la relève en tourisme au Saguenay-Lac-Saint-Jean au cours des vingt dernières années pour dégager l'état de la situation actuelle et faire une projection dans l'avenir.

\section{AVANT LES ANNÉES 1980}

Le Saguenay-Lac-Saint-Jean a connu des heures de gloire et d'exotisme en matière de tourisme. Au tournant du dernier siècle, à l'avènement du chemin de fer à Roberval, la mise en place du complexe Beemer centré sur la pêche du saumon d'eau douce (ouananiche) sur le lac SaintJean a amené jusqu'à mille Américains annuellement. Sur une période un peu plus longue (deux générations et plusieurs miliers de passagers), les grands bateaux blancs de la Canada Steamship Lines ont amené dans le fjord du Saguenay leurs flots de touristes étrangers, à la recherche de sensations fortes, de nature grandiose et sauvage. C'était l'époque des méga projets d'infrastructures industrielles (barrages alimentant des moulins de toutes sortes). À cette époque, les ressources ne pouvaient être que « naturelles". Le contact humain était loin de constituer une composante de l'expérience touristique. La main-d'œuvre était abondante et facilement remplaçable.
Les emplois dans l'industrie touristique étaient des emplois d'exécutants qui pouvaient se comparer à des emplois de domestiques au service de vacanciers qui, pour leur part, ne pouvaient être issus que de l'élite.

Mis à part l'Ermitage Saint-Antoine de Lac-Bouchette, dont le succès immédiat religieuse omniprésente dans la société québécoise durant la période comprise entre les deux guerres, il faut attendre les années 1960 pour observer la démocratisation de l'activité touristique. On assiste alors à l'émergence d'attraits touristiques de masse, ceux-là mêmes qui figurent encore aujourd'hui parmi les principaux générateurs d'achalandage de la région (le Village historique de Val-Jalbert, le Zoo de Saint-Félicien). L'enthousiasme du public dépasse souvent même les espérances des promoteurs. C'est l'époque de la production de masse. Le réseau routier se et constant repose en partie sur la ferveur développe. Une proportion importante des familles possèdent désormais leur automobile, se procurent une tente-roulotte et partent à l'aventure sur les routes de la province, confiantes de pouvoir se loger à faible coût dans l'un ou l'autre des centaines de terrains de camping qui poussent comme des champignons sur le territoire québécois.

Les travailleurs de l'industrie touristique des décennies 1960-1970 et 1970-1980 occupent des fonctions de contrôle des visiteurs, de préposés au stationnement, à la billetterie ou à la crème glacée. C'est le produit qui est au centre de l'expérience touristique. D'ailleurs, c'est le produit qui est l'expérience touristique. L'animation des clientèles des sites touristiques est pratiquement inexistante. Le touriste de cette époque est un « voyeur », il se déplace davantage pour « regarder » que pour « ressentir », ce qui explique en partie le succès immédiat et immense des festivals populaires qui poussent eux aussi comme des champignons et dont la réussite repose sur la popularité des artistes qui figurent au programme. Le produit touristique est consommable tel quel, sur place et sans valeur ajoutée. Les gestionnaires sont plus intéressés à augmenter le nombre de visiteurs qu'à augmenter la durée des séjours. En conséquence, la forte saisonnalité préoccupe les propriétaires quant à la pérennité de leurs équipements. Ceux-ci misent sur le développement du produit et l'innovation pour attirer la clientèle et faire gonfler les entrées au guichet. On est encore très loin à ce moment-là de l'approche «à la Disney » où chaque employé fait partie d'un spectacle (cast member) qui vise à émerveiller l'invité de ce même spectacle (guest). 


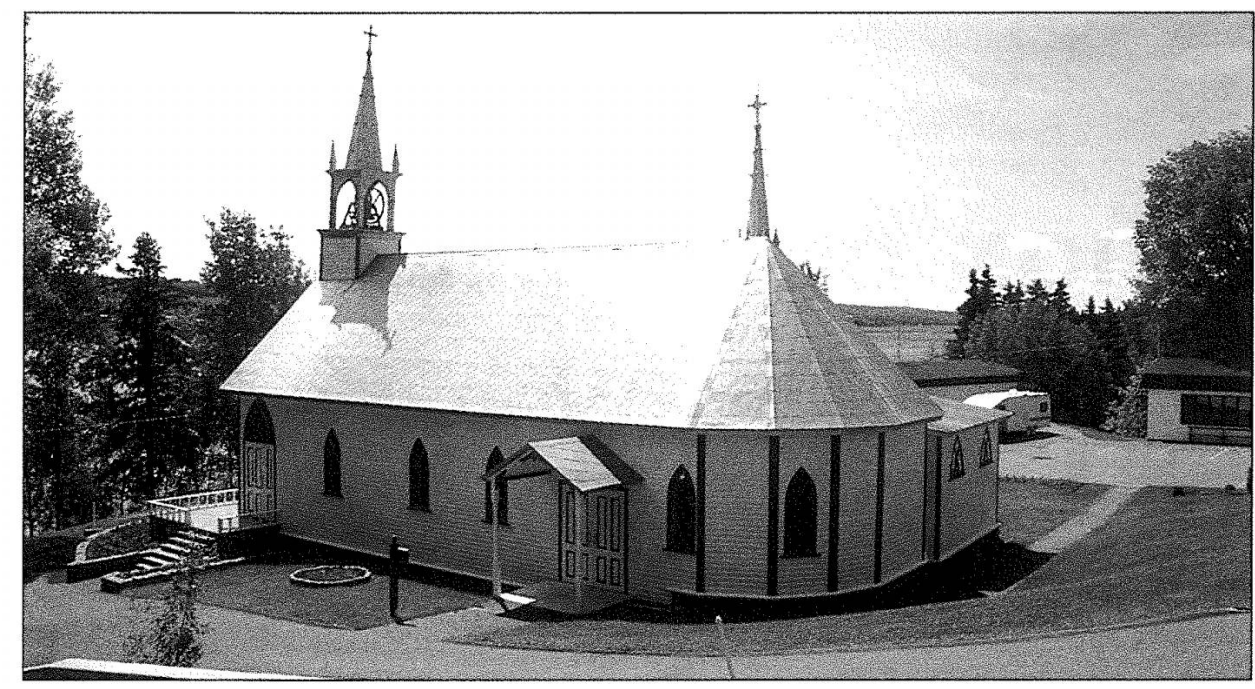

À PARTIR DES ANNÉES 1980

C'est véritablement le début des années 1980 qui verra apparâtre une préoccupation régionale à l'endroit de la formation des ressources humaines comme outil de développement de l'industrie touristique. Jusque-là, les responsables locaux titulaires de permis d'exploitation d'un bureau d'information touristique devaient se rendre au bureau du ministère du Tourisme à Québec pour y apprendre les règles de l'art et les particularités liées à cette fonction essentielle.

En 1980, le Service de la formation continue du Cégep de Saint-Félicien commence à s'intéresser activement au tourisme régional et à mettre en valeur la spécificité du produit touristique de la région. Il prend ainsi la relève du ministère du Tourisme et met sur pied, localement, un cours de formation des préposées et des préposés aux kiosques d'information touristique, en collaboration avec l'Association touristique du SaguenayLac-Saint-Jean. Cette participation et cette collaboration se poursuivent avec succès de façon ininterrompue depuis vingt ans.

À la faveur de la venue de plus en plus fréquente de groupes organisés (clientèles européenne et américaine), une deuxième initiative du Cégep - laquelle se répétera de façon régulière au cours des années ultérieures - consiste en la formation de guides-accompagnateurs touristiques, un programme de 120 heures pour former des guides et des accompagnateurs professionnels aptes autant à travailler en tourisme réceptif sur le plan régional qu'à soutenir le tourisme expéditif.
Mais c'est véritablement la création d'un cours en Accueil touristique adapté au contexte régional qui contribue à former le plus grand nombre de personnes impliquées dans les produits et les services touristiques de toute la région. Au fil des ans, sur l'ensemble du territoire, de Girardville au nord du Lac-Saint-Jean jusqu'à Petit-Saguenay à l'est de la région, c'est plus de mille personnes qui auront profité de cette formation de base, offerte sur place, au sein même de leur entreprise.

Constatant la nécessité de diversifier l'économie touristique du Saguenay-Lac-SaintJean par la création de nouveaux produits et d'activités complémentaires aux produits d'appel, le Cégep de Saint-Félicien met de l'avant, en 1989, un ambitieux projet-pilote d'attestation d'études collégiales (AEC) en entrepreneuriat touristique. De 1989 à 1994, le Service de la formation continue contribue ainsi à la formation d'environ 60 entrepreneurs et gestionnaires en tourisme, contribuant, du même coup, à la création de nouveaux produits. C'est d'ailleurs cette expertise qui a amené le ministère de l'Éducation à accorder au Cégep de Saint-Félicien l'exclusivité de l'attestation d'études collégiales en entrepreneuriat touristique à partir de 1994. Mais les effets les plus intéressants de cette initiative sont sans aucun doute que la région a vu naître un nombre intéressant de nouvelles entreprises et s'améliorer considérablement plusieurs autres, dans à peu près tous les secteurs de l'industrie touristique : hébergement, restauration, activités et attraits, commercialisation, etc.

Le dynamisme démontré par le Service de la formation continue du Cégep de Saint-
Félicien lui a valu d'offrir, dès 1994, le programme de Techniques de tourisme (414.01) à l'enseignement régulier de niveau collégial. Ainsi, le Cégep de SaintFélicien permettait à la clientèle régionale de demeurer en région pour obtenir une formation professionnelle et devenir technicien ou technicienne en tourisme. Quant au ministère de l'Éducation du Québec, il reconnaissait ainsi la qualité du travail de l'institution et la nécessité de la soutenir concrètement dans son projet de contribuer à l'amélioration de l'industrie touristique du Saguenay-Lac-Saint-Jean.

\section{UN PROGRAMME AVEC UNE COULEUR LOCALE}

Bien que légèrement comparable à celui des autres collèges qui dispensent le même programme, celui de Saint-Félicien se démarque par les liens étroits de partenariat qu'il entretient avec l'industrie touristique régionale, québécoise et internationale. L'équipe départementale de Techniques de tourisme du Cégep de Saint-Félicien cumule de nombreuses années d'expérience dans l'industrie touristique québécoise. Cette expérience se transpose non seulement dans les contacts faciles avec les représentants de l'industrie, mais également en activités pédagogiques connectées aux besoins de l'industrie. Ainsi, le plus souvent possible, les travaux exigés des étudiants en tourisme sont ni plus ni moins des mandats réels, des tâches courantes réalisées par des techniciens en poste. Peut-on imaginer des situations de travail plus concrètes que la réalité de l'industrie elle-même ? Organisation d'événements, création de produits, marketing des services, gestion de services d'accueil, animation des clientèles, etc. : toutes ces compétences trouvent un lieu véritable d'expérimentation au sein des entreprises touristiques du Saguenay-Lac-Saint-Jean.

Comme on imagine difficilement que des ressources compétentes ne maîtrisent pas une deuxième, voire une troisième langue, les membres du département consacrent beaucoup d'énergie à accompagner leurs élèves dans la prospection d'un emploi à l'étranger ou dans des milieux propices au perfectionnement linguistique, que ce soit au Canada, aux États-Unis ou ailleurs (Disney World, Disneyland Paris, stage de l'OFQJ, Bunac, Tour du CN, etc.). Il en va ainsi des stages de fin d'études. Malgré le jeune âge du programme à Saint- 
Félicien, déjà une dizaine de participants annuellement vivent des stages d'intégration dans le contexte de la mondialisation, notamment au sein d'entreprises touristiques de l'Amérique du Sud. L'apprentissage de l'espagnol en immersion, l'ouverture à d'autres cultures, le support au développement international dans une approche d'aide humanitaire, amènent les stagiaires à comprendre que le tourisme peut devenir véritablement un instrument de diversification de l'économie et un outil de développement des communautés.

Le Cégep s'est également investi avec succès dans un partenariat un peu plus avancé en assumant la cogestion d'un équipement touristique, en collaboration avec une municipalité. Le bilan actuel de cette initiative est très enthousiasmant, tant au plan des résultats financiers de l'entreprise que des lieux d'observation et d'analyse en classe, de même que des emplois d'été accessibles aux étudiants en tourisme. D'autres ententes en phase d'élaboration avec des communautés locales laissent présager une extraordinaire synergie entre l'institution d'enseignement et son milieu d'appartenance.

\section{LES ENJEUX ET LES PERSPECTIVES}

L'industrie touristique est en constante mutation. Elle évolue au rythme des goûts des consommateurs, obéissant aux modes, aux tendances et aux fluctuations socioéconomico-démographiques. Elle est soumise à de nombreuses lois, dont celle du marché, qui n'est pas la moindre. Elle est aussi tributaire de l'évolution technologique. Mais c'est une industrie prometteuse, car elle procède, elle naît en partie de tous les changements auxquels on assiste. Encore faut-il qu'elle soit à 1'avant-garde, qu'elle anticipe les mouvances, tout en essayant de garder le cap sur l'authenticité, car le produit touristique doit désormais, plus que jamais, comporter une variable associée à l'identité culturelle. Ne parle-ton pas d'ethnotourisme? Par ailleurs, le développement d'un grand nombre d'entreprises touristiques commande également l'élaboration de standards de services ou de standards d'excellence. Il faut aussi encourager l'établissement des seuils d'entrée pour l'industrie, des critères d'embauche qui reconnaîtraient enfin la valeur des techniciens et des techniciennes formés pour assurer la relève. Il s'agit là, à coup sûr, du meilleur moyen pour arriver au professionnalisme attendu de cette industrie et pour faire en sorte que les visiteurs soient vraiment amenés à vivre l'expérience touristique recherchée.

Voilà quelques-uns des éléments qui sont associés à la formation de la relève en tourisme et qui nous interpellent pour amener l'industrie à livrer ses promesses. C'est un défi de taille auquel nous sommes conviés quotidiennement comme institution d'enseignement, mais c'est aussi un défi réalisable et emballant qui, fort heureusement, nous gratifie souvent de résultats spectaculaires associables à l'énergie et aux efforts déployés auprès des jeunes en formation.

Réjean Côté est coordonnateur du département de techniques de tourisme, au Cégep de SaintFélicien.

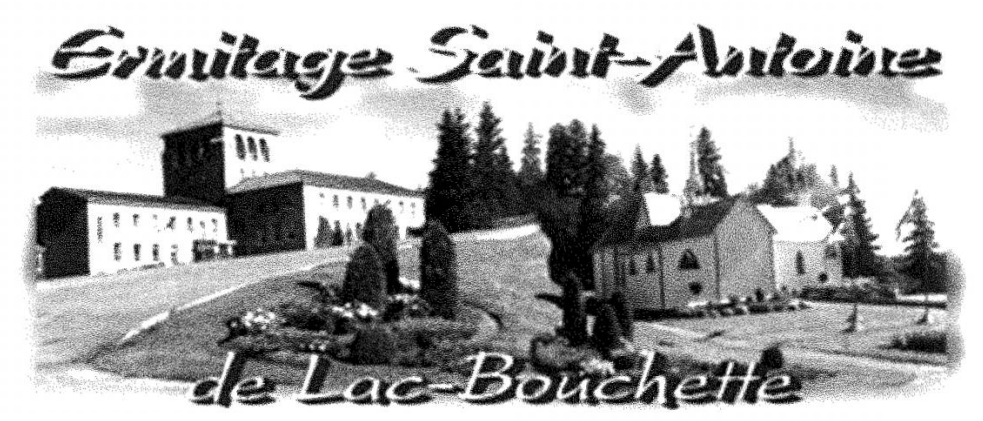

\section{OUVERTURE 07 AVRIL 2000 - - Montée Pascale du 20 avril au 24 avril 2000}

Pour un séjour vraiment inoubliable

La fraternité des pères capucins, l'équipe de pastorale ainsi que tout le personnel de l'Ermitage

Saint-Antoine de Lac-Bouchette vous invitent à profiter de ce havre de paix pour venir vous ressourcer et faire le plein. Laissez ce site enchanteur, situé en pleine nature, vous bercer dans sa beauté, son calme et sa paix.

Forfaits 2000

Occ. Double

Occ. Simple

\begin{tabular}{|c|c|c|c|}
\hline 2 nuits incluant & 2 déjeuners et 3 repas principaux & $78 . \$ /$ pers & $110 . \$ /$ pers \\
\hline 3 nuits incluant & 3 déjeuners et 5 repas principaux & $119 . \$ /$ pers & $166 . \$ /$ pers \\
\hline
\end{tabular}

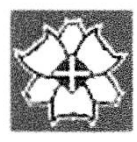

Pour toute information ou pour RÉSERVATIONS

Composez le: 1-800-868-6344

Pour une visite virtuelle: www.destination.ca/ermitage

Ermitage Saint-Antoine de Lac-Bouchette, 250, route de l'Ermitage, Lac-Bouchette, Qc GoW 1V0

Tél: 418-348-6344

Téléc: 418-348-9463 